\title{
A New Method to Study Stochastic Growth Equations: Application to the Edwards-Wilkinson Equation
}

\author{
T. G. Mattos, J. G. Moreira, and A. P. F. Atman \\ Departamento de Física, Instituto de Ciências Exatas, \\ Universidade Federal de Minas Gerais, C.P. 702 30161-123, Belo Horizonte, MG - Brazil
}

Received on 30 September, 2005

\begin{abstract}
In this work we introduce a method to study stochastic growth equations, which follows a dynamics based on cellular automata modeling. The method defines an interface growth process that depends on height differences between neighbors. The growth rules assign a probability $p_{i}(t)$ for site $i$ to receive a particle at time $t$, where $p_{i}(t)=\rho \exp \left[\kappa \Gamma_{i}(t)\right]$. Here $\rho$ and $\kappa$ are two parameters and $\Gamma_{i}(t)$ is a kernel that depends on height $h_{i}(t)$ of site $i$ and on heights of its neighbors, at time $t$. We specify the functional form of this kernel by the discretization of the deterministic part of the equation that describes some growth process. In particular, we study the EdwardsWilkinson (EW) equation which describes growth processes where surface relaxation plays a major role. In this case we have a Laplacian term dominating in the growth equation and $\Gamma_{i}(t)=h_{i+1}(t)+h_{i-1}(t)-2 h_{i}(t)$, which follows from the discretization of $\nabla^{2} h$. By means of simulations and statistical analysis of the height distributions of the profiles, we obtain the roughening exponents, $\beta, \alpha$ and $z$, whose values confirm that the processes defined by the method are indeed in the universality class of the original growth equation.
\end{abstract}

Keywords: Cellular Automata; Interface Growth; Dynamic Scaling

\section{INTRODUCTION}

In nature, as well as in physics laboratories and industrial applications, one can find a large sort of rough surfaces (or interfaces) and the interest in studying such structures has increased in the last decades[1-3]. In a computational approach, one can develop discrete growth models based on a set of symmetries and conservation laws. Proceeding with simulations, one can obtain the scaling exponents and other quantities of interest.

On the other hand, one can write stochastic (continuum) growth equations in order to give an analytical approach to the problem of interface growth, obeying the same symmetries and conservation laws considered in the definition of the mentioned discrete models. Obviously, the agreement of results from both points of view, discrete and continuous, is of fundamental importance.

In the study of interface growth dynamics, one is mostly concerned about the temporal behavior of the interface roughness, which is a measure of the interface width. The most relevant information about the dynamical details of a growth process can be obtained from the temporal behavior of the roughness. In particular, for self-affine interfaces, it is known that the roughness grows with time as a power law, where we define the growth exponent, $\beta$. Actually, due to correlations, the roughness does not grow indefinitely with time; the interface eventually reaches a stationary regime where the roughness saturates. Both the saturation roughness and saturation time depend on the system size as a power law, for which we define the roughness exponent, $\alpha$, and the dynamic exponent, $z$, respectively.

A set of values for these three roughening exponents, in a given dimension, defines an universality class. Thus, if two or more processes have the same exponents values, one can say that they belong to the same universality class, which means that their underlying dynamics obey the same symmetries and conservation laws.

In this work we introduce a method[4] to study stochastic differential equations that describe interface growth processes. This method is based on a cellular automata dynamics[5], in a sense that we associate a particle deposition probability to each site, which depends on the local height profile, considering a synchronous update scheme. Other works that apply cellular automata models to study growth processes have also been done lately[6-8].

Our goal is to show that the method introduced provides the expected results for the universality class of the equation that we are interested in studying. In this paper we apply this method to the Edwards-Wilkinson (EW) equation, which is associated to the random deposition with surface relaxation. In section II we introduce the basic concepts and relevant quantities in the study of interface growth phenomena. In section III we introduce the method and discuss its main features. In section IV we present the simulations results obtained by the application of the method to the EW equation, showing that the method introduced indeed reproduces the expected results for this universality class. Finally, we draw some conclusions and perspectives in section $\mathrm{V}$.

\section{DEFINITIONS}

The discrete computational growth models we are considering in this paper are defined in a one-dimensional lattice of size $L$, initially flat and with periodic boundary conditions. The deposition processes occur in discrete time steps and, in general, we define one time step as the deposition of $L$ particles. The particles are all identical.

In the study of interface growth we are mainly interested in the temporal behavior of the roughness $\omega(L, t)$, which is given by 


$$
\omega^{2}(L, t)=\frac{1}{L} \sum_{i=1}^{L}\left[h_{i}(t)-\bar{h}(L, t)\right]^{2},
$$

where

$$
\bar{h}(L, t)=\frac{1}{L} \sum_{i=1}^{L} h_{i}(t)
$$

is the mean height of the interface.

The roughness provides a measure of the interface width and generally, in growth processes, we have a power law for its temporal behavior,

$$
\omega \sim t^{\beta}
$$

where $\beta$ is the growth exponent. For the simplest deposition model, the random deposition (RD), it is known[2] that the roughness grows indefinitely, with $\beta=1 / 2$. The main characteristic of the RD model is that no correlations are present in the dynamics, hence the collums grow independently from each other.

For other deposition models, where there are correlations among the sites, it is known[2] that the roughness grows initially as the power law (3) and then stabilizes in a value, the saturation roughness $\omega_{\text {sat }}$, after a saturation time $t_{\mathrm{x}}$, for which we have

$$
\begin{gathered}
\omega_{\text {sat }} \sim L^{\alpha}, \\
t_{\mathrm{X}} \sim L^{z},
\end{gathered}
$$

where $\alpha$ is the roughness exponent and $z$ is the dynamic exponent. The three exponents are not independent and, using the Family-Vicsek scaling law[9], it is possible to collapse curves $\omega \times t$ obtained for various system sizes onto a single curve $f(u)$, called scaling function.

Consider for example the random deposition with surface relaxation model (RDSR)[10]. The particle is deposited in a random position in the lattice and is allowed to relax to the position of lowest height, considering the first neighbors. In this way, the particle flux is larger for local minimum than for local maximum positions. For this model the exponent values, in $d=1$, are $[2,10]$

$$
\alpha=\frac{1}{2}, \beta=\frac{1}{4}, z=2 \text {. }
$$

In order to provide an analytical approach to the study of interface growth, one can construct a stochastic growth equation based on the discrete model. However, there is no direct way to derive an equation from the discrete model. All one can do is write down an equation that respects the same symmetries of the discrete model[2] and hope for the best. If the analytical solutions match the previous results obtained from the simulations, one can say that the given equation can be correctly associated to the discrete model.

As we have seen, the particle flux in the RDSR model is larger for local minimum positions. So it is reasonable to say that the time derivative of the local height profile is proportional to the Laplacian. Thus, in order to associate a stochastic equation to this deposition model, we write

$$
\frac{\partial h(\mathbf{x}, t)}{\partial t}=v \nabla^{2} h(\mathbf{x}, t)+\eta(\mathbf{x}, t)
$$

where in the left hand side we have the temporal variation of the height at position $\mathbf{x}$, while in the right hand side we have $v>0$ and $\eta(\mathbf{x}, t)$, which is a white noise (zero average, $\delta$-correlated in space and time). This is the EdwardsWilkinson equation[11], or simply EW equation, which provides the same exponent values obtained for the RDSR model and hence can be correctly associated to this model.

\section{THE METHOD}

Consider a one-dimensional lattice of size $L$, initially flat and with periodic boundary conditions. Each time step, all the sites are simultaneously visited so that site $i$ receives a particle at time $t$ with probability $p_{i}(t)$ given by

$$
p_{i}(t)=\rho e^{\kappa \Gamma_{i}(t)} .
$$

Here $0<\rho<1$ and $\kappa>0$ are two parameters, fixed throughout the evolution of the interface, and $\Gamma_{i}(t)$ is a kernel that depends on the heights of site $i$ and its neighbors. The particular functional form of $\Gamma_{i}(t)$ will be given by the discretization of the deterministic part of the growth equation we are intended to study. In the case of the EW equation (6), the kernel is given by the discretization of the Laplacian $\nabla^{2} h$,

$$
\Gamma_{i}(t)=h_{i+1}(t)+h_{i-1}(t)-2 h_{i}(t) .
$$

In the way we have defined the method, we can eventually get $p_{i}(t)>1$. In this situation, we impose the condition

$$
p_{i}(t) \geq 1 \Longrightarrow p_{i}(t)=1 \Longrightarrow h_{i}(t+1)=h_{i}(t)+1 \text {. }
$$

\section{SIMULATIONS RESULTS}

We applied the method presented in the previous section in the study of the EW equation, with the kernel defined in the expression (8). We initially observed the evolution of the interface for $\rho=0.5, \kappa=0.1$ and $L=500$, as one can see in the top of figure 1 , where we show a typical profile obtained for this set of parameters. In the bottom of figure 1, we show the temporal behavior of the roughness for $\rho=0.5, \kappa=0.1$ and $L=200$. As one can see, the roughness grows initially as a power law with $\beta$ close to $1 / 4$, after a short transient. To 
make sure of the value of the growth exponent, we show in the inset the roughness divided by $t^{1 / 4}$ as a function of $t$, for the same values of the parameters $\rho$ and $\kappa$, but for larger systems $\left(L=10^{2}, 10^{3}\right.$ and $\left.10^{4}\right)$. In this case, a horizontal line means $\beta=1 / 4$ and as one can see, the larger the system size, the closer to $1 / 4$ is the value of the growth exponent, and longer the system stays in this regime.

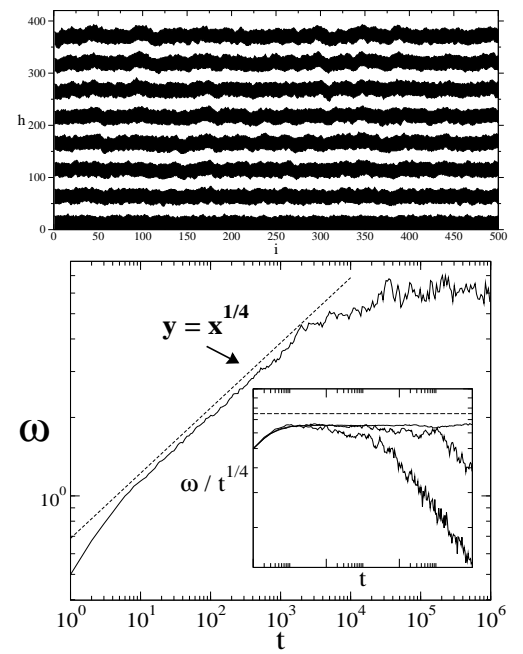

FIG. 1: In the top we show a typical profile for $\rho=0.5, \kappa=0.1$ and $L=500$, where we change the color of the particles each 50 time steps and let the system evolve until $t=750$. In the bottom, we have a log-log plot of the roughness $\omega$ as a function of time $t$, for $\rho=0.5, \kappa=0.1$ and $L=200$, averaged over 200 samples. The traced line corresponds to the function $y=x^{1 / 4}$. In the inset we show the roughness divided by $t^{1 / 4}$ as a function of $t$, for $L=10^{2}, 10^{3}$ and $10^{4}$.

In order to obtain the values of the roughness and dynamic exponents, $\alpha$ and $z$, we let the system reach the saturation regime for some system sizes, between $L=25$ and $L=400$, still keeping $\rho=0.5$ and $\kappa=0.1$, as one can see in figure 2 . In the inset, we show that a good collapse, with $\alpha=1 / 2$ and $z=2$, was obtained, confirming thus that the method, with the kernel given in equation (8), is indeed in the EW universality class.

We then proceed by showing the results obtained by varying the parameter $\kappa$. As one can see in the left of figure 3 , we identified a $\kappa$-dependent crossover between the RD and the RDSR regimes. Initially we have $\beta=1 / 2$ and then $\beta=1 / 4$ and the crossover time $t_{c}$ increases as $\kappa$ decreases. In fact we found a power law for $t_{c}$ as function of $\kappa$,

$$
t_{c} \sim \kappa^{z_{\kappa}^{\prime}}
$$

with $z_{\kappa}^{\prime}=-1.02(2)$, as one can see in the right of figure 3 .

Thus, for small $\kappa$, the system will stay more time in a RD regime with deposition rate $\rho$. In fact, the lower the value of $\kappa$, the higher the value of $\Gamma_{i}(t)$ must be so that $p_{i}(t) \neq \rho$, which

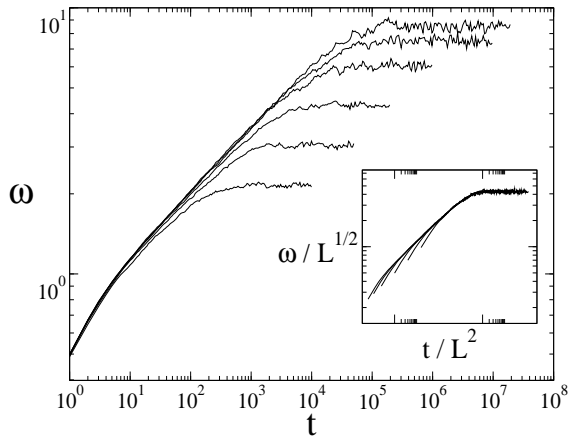

FIG. 2: Keeping $\rho=0.5$ and $\kappa=0.1$, we varied the system size and plotted the roughness $\omega$ against time $t$, for $L=25,50,100,200,300$ and 400 - from bottom to top. In the inset we show the good collapse obtained with $\alpha=1 / 2$ and $z=2$. These graphics are in a log-log scale.
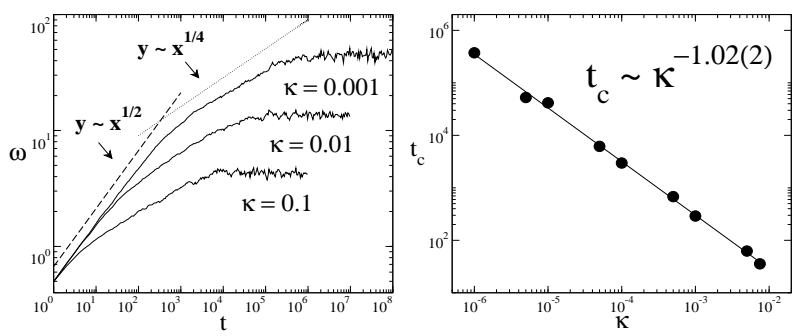

FIG. 3: In the left we have log-log plot of the roughness $\omega$ as a function of time $t$ for $L=250, \rho=0.5$ and $\kappa=10^{-3}, 10^{-2}$ and $10^{-1}$. This result is averaged over 40 samples. Initially we have $\beta=1 / 2$ and then $\beta=1 / 4$, as one can see by comparing with the traced and dotted lines, respectively. In the right, we show the crossover time $t_{c}$ as a function of $\kappa$, for $L=250$ and $\rho=0.5$. For $\kappa>0.02$ it is hard to get precise values for $t_{c}$.

means that the interface roughness must be large enough so that correlations can be seen in the system.

We also studied the dependence of the saturation roughness and saturation time, $\omega_{\text {sat }}$ and $t_{\mathrm{x}}$, with the parameter $\kappa$ and we found that

$$
t_{\mathrm{x}} \sim \kappa^{z \kappa}
$$

$$
\omega_{\text {sat }} \sim \kappa^{\alpha_{\kappa}}
$$

with $\alpha_{\kappa}=-0.511(5)$ and $z_{\kappa}=-1.03(5)$, as one can see in figure 4 .

\section{CONCLUSIONS AND PERSPECTIVES}

We introduced a method to study stochastic growth equations. We applied this method to the study of the EW one-dimensional equation and we found the expected values for the critical exponents within this universality class, 


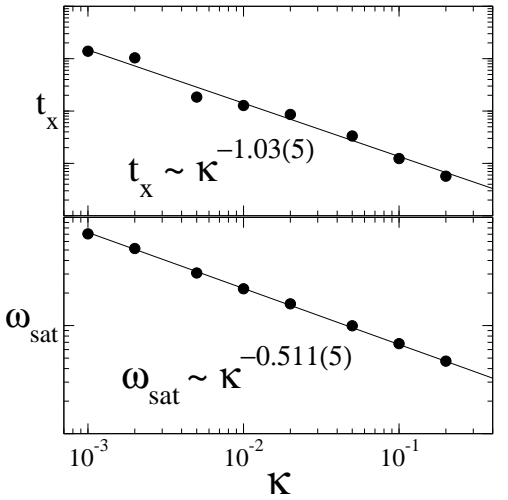

FIG. 4: Log-log plot of the saturation roughness and saturation time, $\omega_{\text {sat }}$ and $t_{\mathrm{x}}$, as functions of the parameter $\kappa$. Here $\rho=0.5, L=250$; the results are averaged over 25 samples. The straight lines are linear fits to the data. proving that the method was successful in this application. We also found a crossover between the RD and RDSR classes when we varied the parameter $\kappa$. A power law behavior was found for the crossover time $t_{c}$, the saturation roughness $\omega_{\text {sat }}$ and the saturation time $t_{\mathrm{x}}$, as functions of $\kappa$. As perspectives we are intended to apply this method in the study of other equations such as the Kardar-Parisi-Zhang equation[12] and the equation of growth with surface diffusion[13, 14]. We shall also apply this method to growth in two-dimensional substrates.

\section{Acknowledgments}

We thank R. Dickman for helpful criticism of the manuscript. This work is supported by Brazilian agency CNPq.
[1] F. Family and T. Vicsek, Dynamics of Fractal Surfaces, World Scientific, Singapore (1991)

[2] A.-L. Barabási and H.E. Stanley, Fractal Concepts in Surface Growth, Cambridge Univ. Press, Cambridge (1995)

[3] P. Meakin, Fractals, scaling and growth far from equilibrium, Cambridge Univ. Press, Cambridge (1998)

[4] T.G. Mattos, "Autômatos celulares e crescimento de interfaces rugosas", Master thesys, UFMG (2005)

[5] S. Wolfram, Theory and Applications of Cellular Automata, World Scientific, Singapore (1986) ; Rev.Mod.Phys. 55, 3, 601 (1983)

[6] A.P.F. Atman, R. Dickman, and J.G. Moreira, Phys.Rev.E 66, $016113(2002)$
[7] A.P.F. Atman and J.G. Moreira, Eur.Phys.J. B 16 (3), 501 (2000)

[8] T.G. Mattos and J.G. Moreira, Brazilian Journal of Physics, 34, 448 (2004)

[9] F. Family and T. Vicsek, J.Phys. A 18, L75-L81 (1985)

[10] F. Family, J.Phys. A 19, L441 (1986)

[11] S.F. Edwards and D.R. Wilkinson, Proc.R.Soc.Lond. A 381, 17$31(1982)$

[12] M. Kardar, G. Parisi, and Y.C. Zhang, Phys.Rev.Lett. 56, 889 (1986)

[13] D.E. Wolf and J. Villain, Europhys. Lett., 13 (5), 389 (1990)

[14] S. Das Sarma and P. Tamborenea, Phys.Rev.Lett. 66, 325 (1991) 